\title{
Stochastic shadowing analysis of a class of stochastic differential equations
}

\author{
Qingyi Zhan ${ }^{\mathrm{a}, \mathrm{b}, *}$, Yuhong $\mathrm{Li}^{\mathrm{c}}$ \\ ${ }^{a}$ College of Computer and Information Science, Fujian Agriculture and Forestry University, Fuzhou, Fujian 350002, P. R. China. \\ ${ }^{b}$ Institute of Computational Mathematics and Scientific/Engineering Computing, Academy of Mathematics and Systems Science, \\ Chinese Academy of Sciences, Beijing 100190, P. R. China. \\ ${ }^{C}$ College of Hydropower and Information Engineering, Huazhong University of Science and Technology, Wuhan, 430074, P. R. China. \\ Communicated by D. Baleanu
}

\begin{abstract}
This paper is devoted to the feasibility of stochastic shadowing of a class of stochastic differential equations via numerical analysis tools. A general shadowing theorem of stochastic differential equations is proven, and an explicit relationship of shadowing and the coefficients of SDE, a bound for shadowing distance are both investigated. The focus is explicit regularity conditions of stochastic differential equations which can ensure the shadowing. A numerical experiment is provided to illustrate the effectiveness of the proposed theorem by the numerical simulations of chaotic orbits of the stochastic Lorenz equations. (C)2017 All rights reserved.
\end{abstract}

Keywords: Stochastic differential equations, random dynamical systems, numerical method, shadowing, multiplicative ergodic theorem, stochastic Lorenz equations. 2010 MSC: 65C20, 65P20, 37H10, 37C50.

\section{Introduction}

In recent times, the shadowing property which is a significant qualitative property of differential equations has played an important role in the theory and application of random dynamical systems (RDS), especially in numerical simulations of chaotic systems of stochastic differential equations (SDEs). Those equations have been applied to describe many natural phenomena, for example in meteorology and biology $[1,10,12,13,18]$. Numerical computations play a significant role in the investigations of the dynamical behavior of chaotic systems of SDEs. In fact, numerical experiments have led to many useful discoveries, such as the discovery of Pluto and reference [14]. However, expecting to find a numerical solution capable of approximating a particular solution of a chaotic system of SDEs for any given length of time is unrealistic, because of the sensitivity of the initial value of the solution and the fact that random noise is constantly being pumped into the system. Nevertheless, the reliability and feasibility of numerical computations have increasingly been attracting attention. This prompts us to investigate whether an approximate numerical solution could be used to imply the dynamics of a chaotic system of SDEs.

\footnotetext{
*Corresponding author

Email addresses: zhanqy@lsec.cc.ac.cn (Qingyi Zhan), liyuhong@hust.edu.cn (Yuhong Li)
} 
This work is motivated by two facts. Firstly, random choice in the deterministic shadowing lemma has been the subject of many studies in terms of the dynamics of random chaotic systems, such as the study performed by Todorov et al. [5, 15] and references therein. In reference [15], relations to stochastic stability and standard shadowing are studied, which are mainly focus on the analytical property of stochastic shadowing. Secondly, the numerical version of shadowing property of random systems, however, has not received as much attention. The constructions of the conditions which can assure the stochastic shadowing in a class of SDEs have been finished in [20,21]. Li and Wang have made useful contributions to the numerical analysis of RDS $[9,16,17]$. These numerical techniques are applied to problems that are hyperbolic, i.e., problems with exponentially stable and unstable components. Shadowing continues to be an interesting method for investigating the dynamical behavior of SDEs. To the best of our knowledge, no investigations of the explicit regularity conditions of SDEs which can ensure stochastic shadowing in a finite-time interval exist in the literature.

Our major contributions are to originate a new approach to investigate the dynamics of chaotic systems of SDEs by numerical calculation, that is, we provide a useful and realizable method for estimating the shadowing distance, i.e., the maximum distance between an $(\omega, \delta)$-pseudo orbit and its corresponding nearest true orbit in mean-square sense. The main difference between existing work and the results presented in this paper is that this work obviates the need to assume hyperbolicity for the original systems, it states explicit regularity assumptions of the SDE that ensures $(\omega, \varepsilon)$-shadowing.

In this work, we utilize the stochastic version of the Newton method to propose a shadowing theorem of SDEs in a finite-time interval, before presenting numerical methods for estimating the shadowing distance of chaotic systems of SDEs. The results show that under certain appropriate assumptions the numerical approximate orbits of discrete approximate RDS are close to the true orbits of the original systems.

The rest of this paper is organized as follows. Section 2 deals with some preliminaries intended to clarify the presentation of concepts and norms used later. In Section 3 the theoretical results of the finite-time shadowing are investigated. Section 4 presents the details of the numerical implementations. Illustrative numerical experiments for the main theorem are included in Section 5. We demonstrate that the main theorem and numerical implementation methods can be applied to the stochastic Lorenz equations (SLEs). Finally, Section 6 is addressed to summarize the conclusions of the paper.

\section{Preliminaries}

Let $(\Omega, \mathcal{F}, \mathbb{P})$ be a canonical Wiener space, $\left\{\mathcal{F}_{t}\right\}_{t \in \mathbb{R}^{+}}$be its natural normal filtration, and $W(t)\left(t \in \mathbb{R}^{+}\right)$ be a standard one-dimensional Brownian motion defined on the space $(\Omega, \mathcal{F}, \mathbb{P})$. And we assume that $\Omega:=\left\{\omega \in \mathbb{C}\left(\mathbb{R}^{+}, \mathbb{R}\right): \omega(0)=0\right\}$, which means that the elements of $\Omega$ can be identified with paths of a Wiener process $\omega(t)=W_{t}(\omega)$. We consider a class of Stratonovich SDEs in the form of

$$
d x_{t}=A x_{t} d t+f_{0}\left(t, x_{t}\right) d t+f_{1} x_{t} \circ d W_{t}, \quad x(s)=x \in \mathbb{R}^{d}, \quad t \geqslant s,
$$

where $A, f_{1}$ are in $\mathcal{L}\left(\mathbb{R}^{d}\right), A$ is a hyperbolic matrix, $f_{0}\left(t, x_{t}\right)$ is Lipschitz continuous in $x_{t}$ so that (2.1) has a unique solution, the random variable $x$ is independent of $\mathcal{F}_{s}$ and satisfies the inequality $\mathbb{E}|x|^{2}<\infty$.

We define

$$
\theta: \mathbb{R}^{+} \times \Omega \rightarrow \Omega, \quad \theta^{t} \omega(s)=\omega(t+s)-\omega(t),
$$

and $0 \leqslant s \leqslant t, s \in \mathbb{R}^{+}, t \in \mathbb{R}^{+}$. It follows from [1, Theorem 2.3.32] that SDE (2.1) generates a unique $\operatorname{RDS} \varphi: \mathbb{R}^{+} \times \mathbb{R}^{+} \times \Omega \times \mathbb{R}^{\mathrm{d}} \rightarrow \mathbb{R}^{\mathrm{d}}$, which is usually written as $\varphi(s, t, \omega) x:=\varphi(s, t, \omega, x)$ on the metric dynamical systems $\left(\Omega, \mathcal{F}, \mathbb{P}, \theta^{t}\right)$, and is $C^{1}$ with respect to $x$, that is, $X_{s, t}^{\chi}(\omega)=\varphi(s, t, \omega, x):=\varphi(s, t, \omega) x$ is a solution to the system of $\operatorname{SDE}(2.1)$ for every $\mathcal{F}_{\mathrm{s}}$-measurable initial data $x \in \mathrm{L}^{2}(\Omega, \mathbb{P})$ at the initial time s. The RDS $\varphi$ is given by

$$
\varphi(s, t, \omega) x=x+\int_{s}^{t}\left[A \varphi(s, r, \omega) x+f_{0}(r, \varphi(s, r, \omega) x)\right] d r+\int_{s}^{t} f_{1} \varphi(s, r, \omega) x \circ d W_{r}(\omega) .
$$


We also make use of the following notations which are similar to $[19,20]$.

- The norm of a random variable $x=\left(x_{1}, x_{2}, \ldots, x_{d}\right) \in \mathrm{L}^{2}(\Omega, \mathbb{P})$ is defined in the form of

$$
\|x\|_{2}=\left[\int_{\Omega}\left[\left|x_{1}(\omega)\right|^{2}+\left|x_{2}(\omega)\right|^{2}+\cdots+\left|x_{d}(\omega)\right|^{2}\right] \mathrm{dP}(\omega)\right]^{\frac{1}{2}}<\infty,
$$

where $\mathrm{L}^{2}(\Omega, \mathbb{P})$ is the space of all square-integrable random variables $x: \Omega \rightarrow \mathbb{R}^{\mathrm{d}}$.

- The norm of a stochastic process $x(t, \omega)$ with $x_{t}(\omega) \in \mathrm{L}^{2}(\Omega, \mathbb{P})$ and $t \in \mathbb{R}^{+}$is defined as

$$
\|x(t, \omega)\|_{2}=\sup _{t \in \mathbb{R}^{+}}\left\|x_{t}(\omega)\right\|_{2}<\infty .
$$

- And the norm of random matrix is defined in the form of

$$
\|A\|_{L^{2}(\Omega, \mathbb{P})}=\left[\mathbb{E}\left(|A|^{2}\right)\right]^{\frac{1}{2}}
$$

where $A$ is a random matrix and $|\cdot|$ is the operator norm.

- For simplicity in notations, the norm $\|\cdot\|_{2}$ and $\|\cdot\|_{\mathrm{L}^{2}(\Omega, \mathbb{P})}$ are usually written as $\|\cdot\|$ unless otherwise stated in sequels.

\section{Theoretical results of stochastic shadowing}

\subsection{Some existed definitions}

Definition 3.1 ([20]). For a given positive number $\delta$ and $\mathbb{P}$-almost surely $\omega \in \Omega$, if there is a sequence of time $\left\{\mathfrak{t}_{k}\right\}_{k=0}^{N}, 0 \leqslant t_{0} \leqslant t_{1} \leqslant \cdots \leqslant t_{N}$ and a sequence of random variables $\left\{\left(u_{k}\left(\theta^{t_{k}} w\right), \mathcal{F}_{t_{k}}\right)\right\}_{k=0}^{N}$ which means that $\mathfrak{u}_{k}\left(\theta^{\mathfrak{t}_{k}} \omega\right)$ is $\mathcal{F}_{\mathfrak{t}_{k}}$-measurable for $k=0,1, \cdots, N$, such that the following inequalities hold

$$
\left\|u_{k+1}\left(\theta^{t_{k+1}} \omega\right)-\varphi\left(t_{k}, t_{k+1}, \theta^{t_{k}} \omega\right) u_{k}\left(\theta^{t_{k}} \omega\right)\right\| \leqslant \delta, \quad k=0,1, \cdots, N-1,
$$

then the random variables $\left\{\left(u_{k}\left(\theta^{t_{k}} \omega\right), \mathcal{F}_{t_{k}}\right)\right\}_{k=0}^{N}$ are said to be an $(\omega, \delta)$-pseudo orbit of SDE (2.1) in the mean-square sense, where $\varphi\left(t_{k}, t_{k+1}, \theta^{t_{k}} \omega\right) u_{k}\left(\theta^{t_{k}} \omega\right)$ denotes the orbit of RDS $\varphi$ at the time $t_{k+1}$ which starts from the initial time $t_{k}$ with the initial value $u_{k}\left(\theta^{t_{k}} \omega\right)$ and the sample $\theta^{t_{k}} \omega$, and $u_{k}\left(\theta^{t_{k}} \omega\right)$ denotes a random variable with the sample $\theta^{t_{k}} \omega$ which is $\mathcal{F}_{t_{k}}$-measurable.

Definition 3.2 ([20]). For a given positive number $\varepsilon, \mathbb{P}$-almost surely $\omega \in \Omega$, and an $(\omega, \delta)$-pseudo orbit $\left\{\left(u_{k}\left(\theta^{t_{k}} w\right), \mathcal{F}_{t_{k}}\right)\right\}_{k=0}^{N}$ of SDE (2.1) with associated times $\left\{t_{k}\right\}_{k=0}^{N}$, if there is a sequence of time

$$
\left\{h_{k}\right\}_{k=0}^{N}, 0 \leqslant h_{0}=t_{0} \leqslant h_{1} \leqslant \cdots \leqslant h_{N},
$$

such that the following inequalities hold

$$
\left\|u_{k}\left(\theta^{t_{k}} \omega\right)-x_{k}\left(\theta^{h_{k}} \omega\right)\right\| \leqslant \varepsilon, \quad 0 \leqslant t_{k}-h_{k} \leqslant \varepsilon, \quad k=0,1, \cdots, N,
$$

where the random variables $\left\{\left(x_{k}\left(\theta^{h_{k}} \omega\right), \mathcal{F}_{h_{k}}\right)\right\}_{k=0}^{N}$ are on the true orbit of SDE (2.1), that is,

$$
x_{k+1}\left(\theta^{h_{k+1}} \omega\right)=\varphi\left(h_{k}, h_{k+1}, \theta^{h_{k}} \omega\right) x_{k}\left(\theta^{h_{k}} \omega\right), \quad k=0,1, \cdots, N-1,
$$

then the $(\omega, \delta)$-pseudo orbit $\left\{\left(u_{k}\left(\theta^{t_{k}} \omega\right), \mathcal{F}_{t_{k}}\right)\right\}_{k=0}^{N}$ is said to be $(\omega, \varepsilon)$-shadowed by a true orbit of SDE (2.1) in the mean-square sense, where the true orbit of $\operatorname{RDS} \varphi$ is a stochastic process.

Definition 3.3 ([20]). The RDS $\varphi: \mathbb{R}^{+} \times \mathbb{R}^{+} \times \Omega \times \mathbb{R}^{\mathrm{d}} \rightarrow \mathbb{R}^{\mathrm{d}}$ is said to be pseudo hyperbolic in mean square if the constants $\kappa_{1}, \kappa_{2} \geqslant 1, v_{1}, v_{2} \geqslant 0$ exist, such that the following inequalities hold with $\mathbb{R}^{\mathrm{d}}=$ $E^{s}(\omega) \oplus E^{\mathfrak{u}}(\omega)$,

$$
\begin{aligned}
& \mathbb{E}\left\|P^{+} \varphi(s, t, \omega) x\right\|^{2} \leqslant \kappa_{1} e^{-v_{1}(t-s)} \mathbb{E}\|x\|^{2}, \forall t \geqslant s, x \in E^{+}(\omega), \\
& \mathbb{E}\left\|P^{-} \varphi(s, t, \omega) x\right\|^{2} \geqslant \kappa_{2} e^{-v_{2}(s-t)} \mathbb{E}\|x\|^{2}, \forall t \geqslant s, x \in E^{-}(\omega),
\end{aligned}
$$

where $P^{ \pm}: \mathbb{R}^{d} \rightarrow E^{ \pm}$be the projection onto $E^{ \pm}$along $E^{\mp}$. 


\subsection{Explicit regularity assumptions of SDE}

This section will provide explicit regularity assumptions of SDE (2.1) that ensure $(\omega, \delta)$-pseudo orbits and $(\omega, \varepsilon)$-shadowing.

Lemma 3.4. Suppose that the matrices $A, A^{*}, f_{1}$ and $f_{1}^{*}$ are mutually commutative, and $\frac{A+A^{*}}{2}$ has only nonzero eigenvalues with the order $\mu_{\mathrm{p}}<\mu_{\mathrm{p}-1}<\cdots<\mu_{\mathrm{m}+1}<\cdots<\mu_{1}, \mathrm{p} \leqslant \mathrm{d}$ and the corresponding eigenspaces $\mathrm{E}_{\mathrm{p}}, \cdots, \mathrm{E}_{1}$ with multiplicity $\mathrm{d}_{\mathrm{i}}=\operatorname{dim}_{\mathrm{i}}$, where $\sum_{i=1}^{\mathrm{p}} \mathrm{d}_{\mathrm{i}}=\mathrm{d}$. And assume that $\mathrm{f}_{0}$ is linear increasing with $\left\|\mathrm{f}_{0}(\mathrm{t}, \mathrm{x})\right\| \leqslant \mathrm{M}_{0}\|x\|$ for $\mathrm{t} \in\left[0, \mathrm{t}_{\mathrm{N}}\right]$ and $x \in \mathbb{R}^{\mathrm{d}}$.

Then the RDS $\varphi$ generated by (2.1) is pseudo hyperbolic.

Proof. It follows from reference [6] that we can define a random evolution operator $\Phi: \mathbb{R}^{+} \rightarrow \mathcal{L}\left(\mathbb{R}^{+}\right)$by

$$
\left\{\begin{array}{l}
\mathrm{d} \Phi_{\mathrm{t}}=A \Phi_{\mathrm{t}} \mathrm{dt}+\mathrm{f}_{1} \Phi_{\mathrm{t}} \circ \mathrm{d} W_{\mathrm{t}}(\omega), \quad \mathrm{t} \geqslant 0, \\
\Phi_{0}=\mathbb{I} \in \mathcal{L}\left(\mathbb{R}^{+}\right) .
\end{array}\right.
$$

Therefore, the solution of (2.1) can be written as follows

$$
\varphi(s, t, \omega) x=\Phi\left(t-s, \theta^{s} \omega\right) x+\int_{s}^{t} \Phi\left(t-r, \theta^{r} \omega\right) f_{0}(r, \varphi(s, r, \omega) x) d r, \quad t \geqslant s,
$$

where the explicit form of $\Phi$ can be provided as

$$
\Phi(t, w)=\exp \left\{A t+f_{1} W_{t}\right\}
$$

Here $E^{-}=E_{p} \oplus E_{p-1} \oplus \cdots \oplus E_{m+1}$ is generated by the eigenvectors with negative eigenvalues, and $\mathrm{E}^{+}=\mathrm{E}_{\mathrm{m}} \oplus \mathrm{E}_{\mathrm{m}-1} \oplus \cdots \oplus \mathrm{E}_{1}$ is generated by the eigenvectors with positive eigenvalues. It follows from (3.1) and [6, Lemma 3.2], we obtain that

$$
\begin{aligned}
\left\|P^{+} \varphi(s, t, \omega) x\right\| & \leqslant\left\|P^{+} \Phi\left(t-s, \theta^{s} \omega\right) x\right\|+\left\|\int_{s}^{t} P^{+} \Phi\left(t-r, \theta^{r} \omega\right) f_{0}(r, \varphi(s, r, \omega) x) d r\right\| \\
& \leqslant\left\|P^{+} \Phi\left(t-s, \theta^{s} \omega\right) x\right\|+M_{0}\left\|\int_{s}^{t} P^{+} \Phi\left(t-r, \theta^{r} \omega\right) d r\right\|\|x\| \\
& \leqslant C_{\Lambda}(\omega) \exp \left(\frac{1}{2} \mu_{m} t\right) \exp (\Lambda|s|)\left[1+M_{0}(t-s)\right]\|x\|,
\end{aligned}
$$

where $\Lambda$ is an arbitrary positive number and $C_{\Lambda}(\omega)$ is a positive random variable depending on $\Lambda$. Similar proof can be done for the case $\left\|\mathrm{P}^{-} \varphi(s, t, \omega) x\right\|$. It follows from Definition 3.3 that this lemma is proved.

This completes the proof of Lemma 3.4.

\subsection{Theoretical analysis of stochastic shadowing}

We wish to show that an $(\omega, \delta)$-pseudo orbit $\left\{\left(y_{k}\left(\theta^{t_{k}} \omega\right), \mathcal{F}_{t_{k}}\right)\right\}_{k=0}^{N}$, which is obtained by one-step numerical methods, is $(\omega, \varepsilon)$-shadowed by a true orbit containing points $\left\{\left(\hat{x}_{k}\left(\theta^{\hat{h}_{k}} \omega\right), \mathcal{F}_{t_{k}}\right)\right\}_{k=0}^{N}$ for $\mathbb{P}$-almost surely $\omega \in \Omega$, that is, we want to find a sequence $\left.\left\{\hat{h}_{k}\right\}\right\}_{k=0}^{N}$, and a sequence of points $\left\{\left(\hat{x}_{k}\left(\theta^{\hat{h}_{k}} \omega\right), \mathcal{F}_{t_{k}}\right)\right\}_{k=0}^{N}$ such that the following inequalities both hold

$$
\left\|\hat{x}_{k}\left(\theta^{\hat{h}_{k}} \omega\right)-y_{k}\left(\theta^{t_{k}} \omega\right)\right\| \leqslant \varepsilon, \quad 0 \leqslant t_{k}-\hat{h}_{k} \leqslant \varepsilon, \quad k=0,1, \cdots, N,
$$

and

$$
\hat{x}_{k+1}\left(\theta^{\hat{h}_{k+1}} \omega\right)=\varphi\left(\hat{h}_{k}, \hat{h}_{k+1}, \theta^{\hat{h}_{k}} \omega\right) \hat{x}_{k}\left(\theta^{\hat{h}_{k}} \omega\right), \quad k=0,1, \cdots, N-1 .
$$


Here we choose a sequence of times $\left\{\hat{h}_{k}\right\}_{k=0}^{N}=\left\{t_{k}\right\}_{k=0}^{N}$ in sequels.

Now we need to introduce a function space $X$ whose element is valued in $\mathbb{R}^{(\mathrm{N}+1) \mathrm{d}}$. Let

$$
y(\omega)=:\left(y_{0}\left(\theta^{t_{0}} \omega\right), y_{1}\left(\theta^{t_{1}} \omega\right), \ldots, y_{N}\left(\theta^{t_{N}} \omega\right)\right),
$$

which is regarded as a special element of the space $X$. And let $x(\omega)=\left(x_{0}\left(\theta^{h_{0}} \omega\right), x_{1}\left(\theta^{h_{1}} \omega\right), \cdots, x_{N}\left(\theta^{h_{N}} \omega\right)\right)$ be a general element of the space $X$ with the norm

$$
\|x(\cdot)\|=\sup _{k}\left\|x_{k}\left(\theta^{h_{k}} \cdot\right)\right\|<+\infty,
$$

where $x(\omega)$ is the symbolic representation of a general point of the space $X$ and

$$
x_{k}\left(\theta^{h_{k}} \omega\right) \in \mathrm{L}^{2}(\Omega, \mathbb{P}) \quad(k=0,1, \cdots, N) .
$$

We consider the mapping $G: X \longrightarrow y$, where $y$ is a function space whose element is valued in $\mathbb{R}^{\mathrm{Nd}}$, and the k-th component of $G(y(\omega))$ is defined for any $y(\omega) \in X$ to be

$$
\begin{aligned}
G(y(\omega))_{k}= & y_{k}\left(\theta^{t_{k}} \omega\right)-\varphi\left(t_{k-1}, t_{k}, \theta^{t_{k-1}} \omega\right) y_{k-1}\left(\theta^{t_{k-1}} \omega\right) \\
= & y_{k}\left(\theta^{t_{k}} \omega\right)-y_{k-1}\left(\theta^{t_{k-1}} \omega\right) \\
& -\int_{t_{k-1}}^{t_{k}} f_{0}\left(\varphi\left(t_{k-1}, s, \theta^{t_{k-1}} \omega\right) y_{k-1}\left(\theta^{t_{k-1}} \omega\right)\right) d s \\
& -\int_{t_{k-1}}^{t_{k}} f_{1}\left(\varphi\left(t_{k-1}, s, \theta^{t_{k-1}} \omega\right) y_{k-1}\left(\theta^{t_{k-1}} \omega\right)\right) \circ d W_{s}(\omega), \quad k=1, \cdots, N,
\end{aligned}
$$

so that $[G(y(\omega))]_{k}$ is the local error at the $k$-th iterate and $y$ is not equal to $X$.

Our purposes are to find a solution

$$
\hat{x}(\omega):=\left(\hat{x}_{0}\left(\theta^{t_{0}} \omega\right), \hat{x}_{1}\left(\theta^{t_{1}} \omega\right), \cdots, \hat{x}_{N}\left(\theta^{t_{N}} \omega\right)\right) \in X, \quad 0 \leqslant t_{k}-\hat{h}_{k} \leqslant \varepsilon,
$$

of the equation $G(\hat{x}(\omega))=0$ and to prove that it lies in a closed $\varepsilon$-neighborhood of $y(\omega)$, where $\left\{\hat{x}_{k}\left(\theta^{\hat{h}_{k}} \omega\right)\right\}$ is $\mathcal{F}_{t_{k}}$-measurable of course.

Here we consider the first variation $\mathrm{DG}(\mathrm{y}(\omega))$ of $\mathrm{G}(\mathrm{y}(\omega))$. By the assumptions that $\operatorname{RDS} \varphi$ generated by SDE (2.1) is $C^{1}$ with respect to $x$, then the Jacobian matrix $D \varphi\left(t_{k-1}, t_{k}, \theta^{t_{k-1}} \omega\right) y_{k-1}$ with respect to $y_{k-1}$ exists. It follows from $G(y(\omega))$ is $C^{1}$ with respect to $y(\omega)$ that the existence of $D G(y(\omega))$ is obtained. Then $\mathrm{DG}(y(\omega))$ is defined by

$$
\begin{aligned}
& \operatorname{DG}(y(\omega))= \\
& \left(\begin{array}{cccccc}
-\mathrm{D} \varphi\left(\mathrm{t}_{0}, \mathrm{t}_{1}, \theta^{\mathrm{t}_{0}} \omega\right) \mathrm{y}_{0} & \mathrm{I} & 0 & \ldots & 0 & 0 \\
0 & -\mathrm{D} \varphi\left(\mathrm{t}_{1}, \mathrm{t}_{2}, \theta^{\mathrm{t}_{1}} \omega\right) \mathrm{y}_{1} & \mathrm{I} & \ldots & 0 & 0 \\
\vdots & \vdots & \vdots & \vdots & \vdots & \vdots \\
0 & 0 & 0 & \ldots & \mathrm{I} & 0 \\
0 & 0 & 0 & \ldots & -\mathrm{D} \varphi\left(\mathrm{t}_{\mathrm{N}-1}, \mathrm{t}_{\mathrm{N}}, \theta^{\mathrm{t}_{\mathrm{N}-1}} \omega\right) \mathrm{y}_{\mathrm{N}-1} & \mathrm{I}
\end{array}\right),
\end{aligned}
$$

where

$$
D \varphi\left(t_{k-1}, t_{k}, \theta^{t_{k-1}} \omega\right) y_{k-1}\left(\theta^{t_{k-1}} \omega\right)=\frac{\partial}{\partial y}\left(\varphi\left(t_{k-1}, t_{k}, \theta^{t_{k-1}} \omega\right) y_{k-1}\left(\theta^{t_{k-1}} \omega\right)\right), \quad k=1,2, \cdots, N,
$$

that is, $\mathrm{DG}(\mathrm{y}(\omega))$ is an $\mathrm{Nd} \times(\mathrm{N}+1) \mathrm{d}$ random matrix, and $\mathrm{I}$ is the $\mathrm{d} \times \mathrm{d}$ identity matrix. Therefore we 
obtain

$[D G(y(\omega)) \Delta y(\omega)]_{k}=\Delta y_{k}\left(\theta^{t_{k}} \omega\right)-D \varphi\left(t_{k-1}, t_{k}, \theta^{t_{k-1}} \omega\right) y_{k-1}\left(\theta^{t_{k-1}} \omega\right) \cdot \Delta y_{k-1}\left(\theta^{t_{k-1}} \omega\right), \quad k=1,2, \cdots, N$, where

$$
\Delta y(\omega)=\left(\Delta y_{0}\left(\theta^{t_{0}} \omega\right), \Delta y_{1}\left(\theta^{t_{1}} \omega\right), \cdots, \Delta y_{N}\left(\theta^{t_{N}} \omega\right)\right)^{\top},
$$

that is, $\Delta y(\omega)$ is the perturbation of $y(\omega)$, and

$$
\Delta y_{k}\left(\theta^{t_{k}} \omega\right)=x_{k}\left(\theta^{t_{k}} \omega\right)-y_{k}\left(\theta^{t_{k}} \omega\right), \quad k=0,1,2, \cdots, N .
$$

Furthermore, we aim to obtain the numerical approximation $L(y(\omega))$ of $D G(y(\omega))$. We assume that $B_{k}\left(\theta^{t_{k}} \omega\right)$ is a $d \times d$ random matrix obtained by numerical integration and satisfies

$$
\left\|B_{k}\left(\theta^{t_{k}} \omega\right)-D \varphi\left(t_{k-1}, t_{k}, \theta^{t_{k-1}} \omega\right) y_{k-1}\left(\theta^{t_{k-1}} \omega\right)\right\| \leqslant \delta
$$

Then $L(y(\omega))$ is given by the expression of DG(y(w)). Therefore we obtain

$$
[\mathrm{L}(\mathrm{y}(\omega)) \Delta \mathrm{y}(\omega)]_{\mathrm{k}}=\Delta \mathrm{y}_{\mathrm{k}}\left(\theta^{\mathrm{t}_{\mathrm{k}}} \omega\right)-\mathrm{B}_{\mathrm{k}}\left(\theta^{\mathrm{t}_{\mathrm{k}}} \omega\right) \Delta \mathrm{y}_{\mathrm{k}-1}\left(\theta^{\mathrm{t}_{\mathrm{k}-1}} \omega\right), \quad \mathrm{k}=1,2, \cdots, \mathrm{N} .
$$

Theorem 3.5. Let $\mathrm{G}: X \longrightarrow \mathrm{y}$ be a $\mathrm{C}^{1}$ map as (3.2) and $\mathrm{L}(\mathrm{y}(\omega))$ be the numerical approximation of $\mathrm{DG}(\mathrm{y}(\omega))$ as (3.3). And let $\mathrm{y}(\omega) \in X$ be a point such that the pseudo inverses $[\mathrm{DG}(\mathrm{y}(\omega))]^{-1}$ and $[\mathrm{L}(\mathrm{y}(\omega))]^{-1}$ exist, and the following inequalities hold

$$
\begin{gathered}
\|\mathrm{G}(\mathrm{y}(\omega))\| \leqslant \delta, \\
\left\|[\mathrm{L}(\mathrm{y}(\omega))]^{-1}\right\| \leqslant \mathrm{c},
\end{gathered}
$$

and

$$
\left\|[\mathrm{L}(\mathrm{y}(\omega))]^{-1}-[\mathrm{DG}(\mathrm{y}(\omega))]^{-1}\right\| \leqslant \alpha,
$$

for some positive constants $\delta, \mathrm{c}$ and $\alpha$. Furthermore, we let $\varepsilon:=2(\alpha+\mathrm{c}) \delta$. If the inequality

$$
\|\mathrm{DG}(\hat{x}(\omega))-\mathrm{L}(\mathrm{y}(\omega))\|=\beta \leqslant \frac{1}{2(\alpha+\mathrm{c})},
$$

holds for $\|\hat{x}(\omega)-y(\omega)\| \leqslant \varepsilon$, then the equation $G(\hat{x}(\omega))=0$ has one solution $\hat{x}(\omega)$ which satisfies

$$
\|\hat{x}(\omega)-y(\omega)\| \leqslant \varepsilon
$$

Proof. For the proof this is immediate from the stochastic version of the Newton method [8].

In order to prove the solvability of the equation $G(\hat{x}(\omega))=0$, we first construct a contracting mapping of $\hat{x}(\omega)$. It follows from the Taylor expansion of vector function [8] that

$$
\begin{aligned}
G(\hat{x}(\omega)) & =G(y(\omega))+D G(y(\omega))(\hat{x}(\omega)-y(\omega))+o(|\hat{x}(\omega)-y(\omega)|) \\
& =G(y(\omega))+L(y(\omega))(\hat{x}(\omega)-y(\omega))+o(|\hat{x}(\omega)-y(\omega)|)
\end{aligned}
$$

that is, $\mathrm{DG}(\mathrm{y}(\omega))$ is approximated by $\mathrm{L}(\mathrm{y}(\omega))$ and the error is taken into the last term of (3.7). If $\mathrm{G}(\hat{x}(\omega))=0,(3.7)$ implies that

$$
\hat{x}(\omega)=y(\omega)-[L(y(\omega))]^{-1} \cdot[G(y(\omega))+o(|\hat{x}(\omega)-y(\omega)|)]:=T(\hat{x}(\omega)) .
$$

Secondly, we prove the mapping $T(\hat{x}(\omega))$ is contracting. If arbitrary $x_{j_{1}}(\omega) \in X$ and $x_{j_{2}}(\omega) \in X$ satisfy

$$
\left\|x_{j_{1}}(\omega)-y(\omega)\right\| \leqslant \varepsilon,\left\|x_{j_{2}}(\omega)-y(\omega)\right\| \leqslant \varepsilon,
$$


we have

$$
\begin{aligned}
\left\|o\left(\left|x_{j_{1}}(\omega)-y(\omega)\right|\right)-o\left(\left|x_{j_{2}}(\omega)-y(\omega)\right|\right)\right\| \leqslant & \left\|x_{j_{1}}(\omega)-x_{j_{2}}(\omega)\right\| \\
& \times \int_{0}^{1}\left\|\left[D G\left[x_{j_{2}}(\omega)+\lambda\left(x_{j_{1}}(\omega)-x_{j_{2}}(\omega)\right)\right]-L(y(\omega))\right]\right\| d \lambda .
\end{aligned}
$$

It follows from (3.5) and the inequality properties of the norm that

$$
\left\|[\operatorname{DG}(y(\omega))]^{-1}\right\|-\left\|[\mathrm{L}(y(\omega))]^{-1}\right\| \leqslant\left\|[\mathrm{DG}(y(\omega))]^{-1}-[\mathrm{L}(\mathrm{y}(\omega))]^{-1}\right\| \leqslant \alpha .
$$

Therefore, we have

$$
0<\left\|[\mathrm{DG}(\mathrm{y}(\omega))]^{-1}\right\| \leqslant \alpha+\mathrm{c} .
$$

Note that $\delta=\frac{\varepsilon}{2(\alpha+c)}$ and (3.8), we have $\delta \leqslant \frac{\varepsilon}{2\left\|[\mathrm{DG}(\mathrm{y}(\boldsymbol{w}))]^{-1}\right\|}$. Following from (3.2) and the definition of $(\omega, \delta)$-pseudo orbit, we have

$$
\|\mathrm{G}(\mathrm{y}(\omega))\| \leqslant \delta \leqslant \frac{\varepsilon}{2\left\|[\mathrm{DG}(\mathrm{y}(\omega))]^{-1}\right\|}
$$

Moreover, utilizing (3.8), we obtain

$$
0<\frac{1}{2(\alpha+c)} \leqslant \frac{1}{2\left\|[\mathrm{DG}(\mathrm{y}(\omega))]^{-1}\right\|} .
$$

From (3.6) we have the following estimate

$$
\|\mathrm{DG}(\hat{x}(\omega))-\mathrm{L}(\mathrm{y}(\omega))\| \leqslant \frac{1}{2(\alpha+c)} \leqslant \frac{1}{2\left\|[\mathrm{DG}(\mathrm{y}(\omega))]^{-1}\right\|} .
$$

It follows from (3.8) and (3.9) that we can obtian the following estimate

$$
\left\|o\left(\left|x_{j_{1}}(\omega)-y(\omega)\right|\right)-o\left(\left|x_{j_{2}}(\omega)-y(\omega)\right|\right)\right\| \leqslant\left\|x_{j_{1}}(\omega)-x_{j_{2}}(\omega)\right\| \cdot \frac{1}{2\left\|[\operatorname{DG}(y(\omega))]^{-1}\right\|} .
$$

Let $\mathcal{B}_{\varepsilon}=\{\hat{x}(\omega) \in X,\|\hat{x}(\omega)-y(\omega)\| \leqslant \varepsilon\}$. Now our goal is to prove that $T$ is a contracting mapping on $\mathcal{B}_{\varepsilon}$. If $\hat{x}(\omega) \in \mathcal{B}_{\varepsilon}$, this implies

$$
\|\mathrm{T}(\hat{x}(\omega))-y(\omega)\| \leqslant\left\|[\mathrm{L}(\mathrm{y}(\omega))]^{-1}\right\| \cdot[\|\mathrm{G}(\mathrm{y}(\omega))\|+\|\mathrm{o}(|\hat{x}(\omega)-y(\omega)|)\|] .
$$

It follows from (3.7) and (3.10) that

$$
\|o(|\hat{x}(\omega)-y(\omega)|)\| \leqslant\|\hat{x}(\omega)-y(\omega)\| \frac{1}{2\left\|[\operatorname{DG}(y(\omega))]^{-1}\right\|} .
$$

Then it follows from (3.8) and (3.11) that

$$
\|\mathrm{T}(\hat{x}(\omega))-y(\omega)\| \leqslant\left\|[\mathrm{L}(\mathrm{y}(\omega))]^{-1}\right\| \cdot\left[\frac{\varepsilon}{2\left\|[\mathrm{DG}(\mathrm{y}(\omega))]^{-1}\right\|}+\|\hat{x}(\omega)-y(\omega)\| \frac{1}{2\left\|[\mathrm{DG}(y(\omega))]^{-1}\right\|}\right] \leqslant \varepsilon .
$$

This shows that the mapping $\mathrm{T}$ maps $\mathcal{B}_{\varepsilon}$ into itself.

If arbitrary $x_{j_{1}}(\omega), x_{j_{2}}(\omega) \in \mathcal{B}_{\varepsilon}$, the inequality (3.10) implies that

$$
\left\|T\left(x_{j_{1}}(\omega)\right)-T\left(x_{j_{2}}(\omega)\right)\right\| \leqslant \frac{1}{2}\left\|x_{j_{1}}(\omega)-x_{j_{2}}(\omega)\right\| \leqslant \frac{\varepsilon}{2} .
$$

Therefore, $\mathrm{T}$ is a contracting mapping on $\mathcal{B}_{\varepsilon}$.

Last but not least, it follows from $T$ is a contracting mapping that there exists a point $\hat{x}(\omega) \in \mathcal{B}_{\varepsilon}$ such that $T(\hat{x}(\omega))=0$. Then by (3.7) the equation $G(\hat{x}(\omega))=0$ has one solution $\hat{x}(\omega) \in \mathcal{B}_{\varepsilon}$. The proof is finished. 
Remark 3.6. As we see, Theorem 3.5 is an approximate random implicit function theorem, that is, we can use it to find a solution of the equation $\mathrm{G}(\hat{\mathrm{x}}(\omega))=0$. Meanwhile, it is a shadowing type theorem which we use in sequels to show that there exists a point $\hat{x}(\omega) \in \mathcal{X}$ on the true solution in the neighbourhood of which we compute by numerical methods.

Theorem 3.7. Let $\left\{\left(\mathrm{y}_{\mathrm{k}}\left(\theta^{\mathrm{t}_{\mathrm{k}}} \omega\right), \mathcal{F}_{\mathrm{t}_{\mathrm{k}}}\right)\right\}_{\mathrm{k}=0}^{\mathrm{N}}$ be an $(\omega, \delta)$-pseudo orbit of SDE (2.1) obtained by one-step scheme and satisfies (3.6). And suppose RDS $\varphi$ generated by SDE (2.1) satisfies Lemma 3.4. For a given sufficiently small $\varepsilon>0$, there exists a positive constant $\delta>0$ such that the $(\omega, \delta)$-pseudo orbit $\left\{\left(y_{k}\left(\theta^{t_{k}} \omega\right), \mathcal{F}_{t_{k}}\right)\right\}_{k=0}^{N}$ is $(\omega, \varepsilon)$-shadowed by a true orbit containing points $\left\{\left(\hat{x}_{k}\left(\theta^{\hat{h}_{k}} \omega\right), \mathcal{F}_{t_{k}}\right)\right\}_{k=0}^{N}$, where $\left\{\hat{h}_{k}\right\}_{k=0}^{N}=\left\{t_{k}\right\}_{k=0}^{N}$. Moreover, the shadowing distance is $\varepsilon=2(\alpha+c) \delta$.

Proof. Given an $(\omega, \delta)$-pseudo orbit $\left\{\left(y_{k}\left(\theta^{t_{k}} \omega\right), \mathcal{F}_{t_{k}}\right)\right\}_{k=0}^{N}$, let

$$
y(\omega):=\left(y_{0}\left(\theta^{t_{0}} \omega\right), y_{1}\left(\theta^{t_{1}} \omega\right), \cdots, y_{N}\left(\theta^{t_{N}} \omega\right)\right),
$$

be a point of the space $X$. We wish to show that $y(\omega)$ is $(\omega, \varepsilon)$-shadowed by a true orbit containing points $\left\{\left(\hat{x}_{k}\left(\theta^{\hat{h}_{k}} \omega\right), \mathcal{F}_{t_{k}}\right)\right\}_{k=0}^{N}$. Here we choose a sequence of times $\left\{\hat{h}_{k}\right\}_{k=0}^{N}=\left\{t_{k}\right\}_{k=0}^{N}$ in sequels so that $\hat{x}_{k}\left(\theta^{\hat{h}_{k}} \omega\right)$ is $\mathcal{F}_{t_{k}}$-measurable. It is transformed to find a solution

$$
\hat{x}(\omega):=\left(\hat{x}_{0}\left(\theta^{t_{0}} \omega\right), \hat{x}_{1}\left(\theta^{t_{1}} \omega\right), \cdots, \hat{x}_{N}\left(\theta^{t_{N}} \omega\right)\right) \in X,
$$

of the equation $G(\hat{x}(\omega))=0$ which lies in a closed $\varepsilon$-neighborhood of $y(\omega)$.

Now we only need to check that the requirements of Theorem 3.5 are satisfied by Theorem 3.7.

First of all, it follows from the definitions of $(\omega, \delta)$-pseudo orbit and the mapping $G$ that the inequality $\|\mathrm{G}(\mathrm{y}(\omega))\| \leqslant \delta$ holds.

Secondly, we will prove that the pseudo inverses $[\mathrm{DG}(\mathrm{y}(\omega))]^{-1}$ and $[\mathrm{L}(\mathrm{y}(\omega))]^{-1}$ exist. It follows from the assumption that $\operatorname{RDS} \varphi$ has pseudo hyperbolicity in mean square sense in the interval $\left[0, t_{N}\right]$. By the stochastic Hartman-Grobman theorem [4], RDS $\varphi$ in $\left[0, t_{N}\right]$ is locally topologically conjugate to the $(\omega, \delta)$-pseudo orbit $\left\{\left(y_{k}\left(\theta^{t_{k}} \omega\right), \mathcal{F}_{t_{k}}\right)\right\}_{k=0}^{N}$ for $\mathbb{P}$-almost surely $\omega \in \Omega$. It is obvious that

$$
\varphi\left(t_{k}, t_{k+1}, \theta^{t_{k}} \omega\right) \cdot: \mathbb{B} \rightarrow \mathbb{R}^{d}, \quad k=0,1, \cdots, N,
$$

can be viewed as a sequence of mappings, and the random difference equation

$$
v_{k+1}=D \varphi\left(t_{k}, t_{k+1}, \theta^{t_{k}} \omega\right) y_{k}\left(\theta^{t_{k}} \omega\right) \cdot v_{k}, \quad k=0,1, \cdots, N,
$$

has exponential dichotomy in mean-square sense [3, Definition 5.2.2], where $v_{k} \in \mathbb{R}^{\mathrm{d}}$. Then it follows from the roughness theorem [3, Theorem 5.2.4] that

$$
v_{k+1}=D \varphi\left(t_{k}, t_{k+1}, \theta^{t_{k}} \omega\right) y_{k}\left(\theta^{t_{k}} \omega\right) \cdot v_{k}, \quad k=0,1, \cdots, N,
$$

has exponential dichotomy in mean-square sense, too. Then we obtain that

$$
\operatorname{det}\left(D \varphi\left(t_{k}, t_{k+1}, \theta^{t_{k}} \omega\right) y_{k}\left(\theta^{t_{k}} \omega\right)\right) \neq 0,
$$

i.e., the pseudo inverses

$$
\left(D \varphi\left(t_{k}, t_{k+1}, \theta^{t_{k}} \omega\right) y_{k}\left(\theta^{t_{k}} \omega\right)\right)^{-1}, \quad k=0,1, \cdots, N,
$$

exists a.s.

By the determinant calculation, we can obtain that

$$
\operatorname{det}(\mathrm{DG}(\mathrm{y}(\omega))) \neq 0 \text {. }
$$

Therefore, the pseudo inverse $[\mathrm{DG}(\mathrm{y}(\omega))]^{-1}$ exists a.s. Because $\mathrm{B}_{\mathrm{k}}\left(\theta^{t_{k}} \omega\right)$ is the numerical approximation 
of $D \varphi\left(t_{k}, t_{k+1}, \theta^{t_{k}} \omega\right) y_{k}\left(\theta^{t_{k}} \omega\right)$, the pseudo inverses

$$
\left(B_{k}\left(\theta^{t_{k}} \omega\right)\right)^{-1}, \quad k=0,1, \cdots, N,
$$

exist a.s. Therefore, $[\mathrm{L}(\mathrm{y}(\omega))]^{-1}$ exists by the determinant calculation.

Note that $[\mathrm{DG}(\mathrm{y}(\omega))][\mathrm{DG}(\mathrm{y}(\omega))]^{\top}$ is a sparse random matrix with a block-tridiagonal structure, then the Moore-Penrose inverse $[\mathrm{DG}(\mathrm{y}(\omega))]^{-1}$ can be efficiently computed as

$$
[\mathrm{DG}(\mathrm{y}(\omega))]^{-1}=[\mathrm{DG}(\mathrm{y}(\omega))]^{\mathrm{T}}\left([\mathrm{DG}(\mathrm{y}(\omega))] \cdot[\mathrm{DG}(\mathrm{y}(\omega))]^{\mathrm{T}}\right)^{-1},
$$

and the norm $\left\|[\mathrm{DG}(\mathrm{y}(\omega))]^{-1}\right\|$ is bounded for $\mathbb{P}$-almost surely $\omega$.

Last but not least, we will show the existence of $c$ as follows. By the QR decomposition, we obtain $\mathrm{L}(\mathrm{y}(\omega))=\mathrm{Q}(\omega) \cdot \mathrm{R}(\omega)$, where $\mathrm{Q}(\omega)$ is a random orthogonal matrix and $\mathrm{R}(\omega)$ is a random upper triangular matrix. Therefore, we obtain

$$
[\mathrm{L}(\mathrm{y}(\omega))]^{-1}=[\mathrm{R}(\omega)]^{-1} \cdot \mathrm{Q}(\omega)^{\mathrm{T}} .
$$

Then we can find a constant $\mathrm{c}$ such that $\left\|[\mathrm{L}(\mathrm{y}(\omega))]^{-1}\right\| \leqslant \mathrm{c}$. So the inequality (3.4) holds. Similarly, we can calculate $[\mathrm{DG}(\mathrm{y}(\omega))]^{-1}-[\mathrm{L}(\mathrm{y}(\omega))]^{-1}$, then we deduce that the inequality (3.5) holds by the same method.

Therefore, it follows from Theorem 3.5 that there is a solution $\hat{x}(\omega)$ of the equation $G(\hat{x}(\omega))=0$ which lies in a closed $\varepsilon$-neighbourhood of $y(\omega)$. That is, the solution $\hat{x}(\omega)$ obtained from Theorem 3.5 is indeed a solution of this problem. Then the shadowing distance is $\varepsilon=2(\alpha+c) \delta$. This completes the proof.

Remark 3.8. Theorem 3.7 provides an explicit relationship between the $(\omega, \varepsilon)$-shadowing and the coefficients of SDE (2.1). This leads to a great deal of conveniences that we omit the calculation of some complex coefficients.

\section{Numerical implementation methods}

In this section, we approximate the local error $\delta$ using the local error control mechanism of the numerical scheme. Then we only pay attention to the magnification of the local error, $(\alpha+c)$, that gives the shadowing distance.

Step 1. Utilizing the one-step numerical scheme (EM scheme or Milstein scheme [11]) to simultaneously solve the following equations from $t_{k}$ to $t_{k+1}$ with the initial values $x(0)=y_{k}\left(\theta^{t_{k}} \omega\right)$ and $v(0)=I$,

$$
\left\{\begin{array}{l}
d x_{t}=A x_{t} d t+f_{0}\left(t, x_{t}\right) d t+f_{1} x_{t} \circ d W_{t}(\omega), \\
d v_{t}=\left(A+D f_{0}\left(t, x_{t}\right)\right) v_{t} d t+f_{1} v_{t} \circ d W_{t}(\omega),
\end{array}\right.
$$

then we obtain the approximations of $\hat{x}_{k+1}\left(\theta^{t_{k+1}} \omega\right)$ and $D \varphi\left(t_{k}, t_{k+1}, \theta^{t_{k}} \omega\right) y_{k}\left(\theta^{t_{k}} \omega\right)$, respectively [7]

$$
\begin{gathered}
\hat{x}_{k+1}\left(\theta^{t_{k+1}} \omega\right) \approx \varphi\left(t_{k}, t_{k+1}, \theta^{t_{k}} \omega\right) y_{k}\left(\theta^{t_{k}} \omega\right), \\
D \varphi\left(t_{k}, t_{k+1}, \theta^{t_{k}} \omega\right) y_{k}\left(\theta^{t_{k}} \omega\right) \approx v_{k+1}\left(\theta^{t_{k+1}} \omega\right)=B_{k+1}\left(\theta^{t_{k+1}} \omega\right) .
\end{gathered}
$$

Step 2. It follows from the oscillation theorem of linear equations that we can find $\alpha$ such that

$$
\left\|[\mathrm{L}(\mathrm{y}(\omega))]^{-1}-[\mathrm{DG}(\mathrm{y}(\omega))]^{-1}\right\| \leqslant \alpha .
$$

Step 3. By the norm properties, we can find $\beta$ such that

$$
\beta=\|D G(\hat{x}(\omega))-L(y(\omega))\| .
$$

Step 4. If the conditions of Theorem 3.7 are satisfied, the values of $c, \alpha, \delta$ and $\beta$ satisfy all conditions of Theorem 3.5, too. Therefore, the shadowing distance is $\varepsilon=2(c+\alpha) \delta$. In sequels we choose $\hat{h}_{k}=t_{k}, k=$ $0,1, \cdots, \mathrm{N}$. 


\section{An example}

This section will provide numerical experiments to test the conclusion of Theorem 3.7.

\subsection{Experimental preparation}

We consider the Stratonovich SLEs

$$
d X_{t}=A X_{t} d t+f_{0}\left(t, X_{t}\right) d t+f_{1} X_{t} \circ d W_{t}(\omega), \quad X(0)=x_{0} \in \mathbb{R}^{3},
$$

where $X_{t}=(x, y, z)^{\top} \in \mathbb{R}^{3}, x, y$ and $z$ make up the system state, $\sigma, \rho$ and $\beta$ are the system parameters, and

$$
A=\left(\begin{array}{ccc}
-\sigma & \sigma & 0 \\
\rho & -1 & 0 \\
0 & 0 & -\beta
\end{array}\right), \quad f_{0}\left(t, X_{t}\right)=\left(\begin{array}{c}
0 \\
-x z \\
x y
\end{array}\right), \quad f_{1} X_{t}=\left(\begin{array}{c}
\mu x \\
\mu y \\
\mu z
\end{array}\right) .
$$

In this experiment, we choose the classic parameters $\sigma=10, \rho=28, \beta=\frac{8}{3}$. It is the fact that these parameters satisfy Theorem 3.7.

The existence and uniqueness of solution of SDE (5.1) can be proved by the same approaches as proposed in [1] though a normally required linear growth condition is not satisfied. Hence, an $\operatorname{RDS}(\varphi, \theta)$ can be generated by the solution operator of SDE (5.1).

It follows from [2] that a global attractor, i.e., a forward invariant random compact set of $\operatorname{RDS} \varphi$ generated by SDE (5.1) is the closed ball $\mathbb{B}_{1}$ with center zero and radius $\mathcal{R}(\omega)$, that is,

$$
\mathbb{B}_{1}=\left\{X_{t} \in \mathbb{R}^{3}:\left\|X_{t}\right\| \leqslant \mathcal{R}(\omega)\right\}
$$

where

$$
\mathcal{R}(\omega)=c_{2} \int_{-t_{N}}^{0} \exp \left(c_{1} s-2 \sigma W_{s}(\omega)\right) d s
$$

and

$$
\begin{gathered}
c_{1}=\min (1, \beta, \sigma), \quad c_{2}>0, \quad 2\langle B u, u\rangle<-c_{1}|u|^{2}+c_{2}, \\
B=\left(\begin{array}{ccc}
-\sigma & \sigma & 0 \\
\rho & -1 & 0 \\
0 & 0 & -\beta
\end{array}\right) .
\end{gathered}
$$

The preset initial conditions are as follows: the initial value is $x_{0}=0, y_{0}=1, z_{0}=0$. Here we take the temporal step-size $\Delta t=7.0 e-3$, the iterative step $N=1.5 e+4$ and $\mu=0.5$. SDE (5.1) is discretized by Euler-Maruyama (EM) scheme, we obtain that

$$
\left\{\begin{array}{l}
x_{k+1}=x_{k}+\left(\sigma\left(-x_{k}+y_{k}\right)+0.5 \mu^{2} x_{k}\right) \Delta t_{k}+\mu x_{k} \Delta W_{k} \\
y_{k+1}=y_{k}+\left(-x_{k} z_{k}-\rho x_{k}+y_{k}+0.5 \mu^{2} y_{k}\right) \Delta t_{k}+\mu y_{k} \Delta W_{k}, \\
z_{k+1}=z_{k}+\left(x_{k} y_{k}-\beta z_{k}+0.5 \mu^{2} z_{k}\right) \Delta t_{k}+\mu z_{k} \Delta W_{k} .
\end{array}\right.
$$

It follows that SDE (5.1) with the classic parameters possesses a one point random attractor, i.e., a unique random fixed point $\bar{X}(\omega)$, where

$$
\bar{X}(\omega)=\lim _{k \rightarrow+\infty}\left(x_{k}, y_{k}, z_{k}\right)=(0,0,0),
$$

which is obtained by the rigorous numerical computation. This global attractor is shown in Figure 1. As we can see, with the increase of the iterative step $n$, the orbit tends to a fixed area. That is, after the initial finite iterative step $n$, it looks like a cylinder. Moreover, Figure 1 is a special case of the experiments which are shown as Figure 2 . Therefore, $\mathbb{B}_{1}$ is attracting. This shows that there exists a forward invariant random compact set for SLEs. 


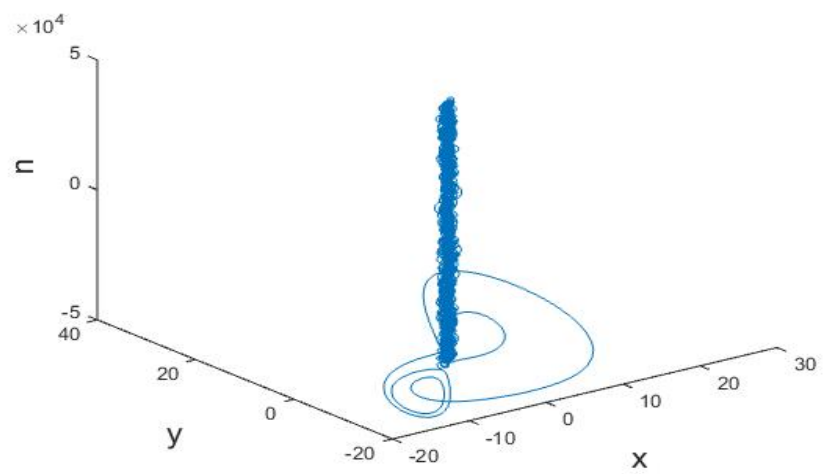

Figure 1: An orbit of length 100000 on the stochastic Lorenz attractor, plotted over its index n.

\subsection{Numerical results in detail}

This section will provide numerical experiments to compute the shadowing distance of SDE (5.1). The classical parameters are chosen and the initial value is $x_{0}=0, y_{0}=1, z_{0}=0$.

Firstly, in order to show the influence of noise on the pseudo orbits, we choose various size of noise, such as $\mu=0$ (the deterministic case), $\mu=0.5, \mu=1.0$ and $\mu=1.35$. And we take the temporal step-size $\Delta t=1.05 e-3$ and $N=1.0 e+4$. Taking the numerical solution for an example, Figure 2 shows the perturbation of the pseudo orbits of SDE (5.1) by using Method I corresponding to differential scales of noise. It shows that the perturbation of the pseudo orbits becomes much more seriously both in $x$ and $y$ directions due to the increases of the scale of the noise.
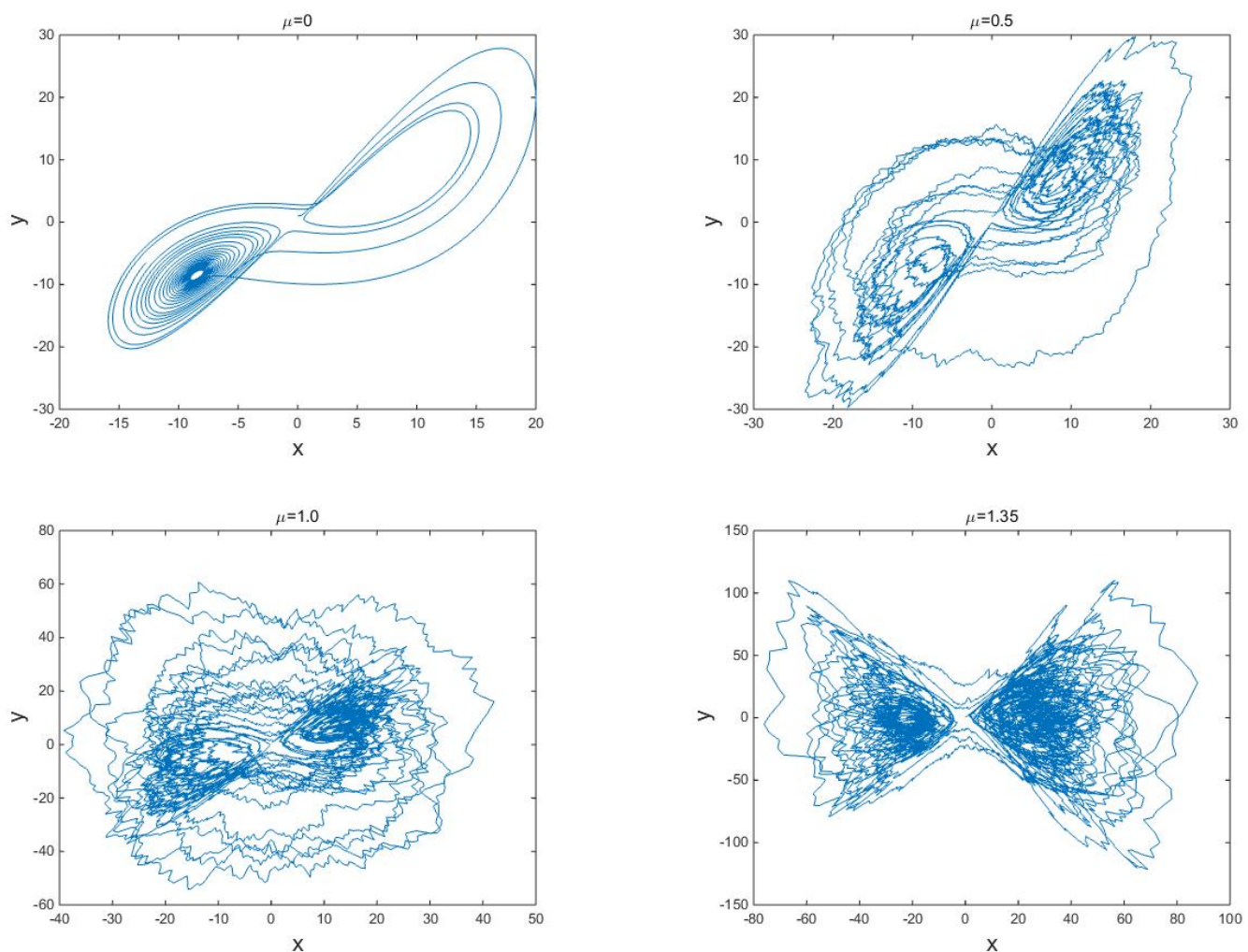

Figure 2: Experimental results of pseudo orbits for different sizes of noise $\mu=0, \mu=0.5, \mu=1.0$ and $\mu=1.35$. 
Secondly, we focus on the numerically performing of the shadowing distance $\varepsilon=2(\alpha+c) \delta$. The amplification factors $\alpha$ and $c$ are computed quite accurately, and the local error tolerance $\delta$ is approximately determined by the numerical scheme. Applying EM scheme and Milstein scheme to SLEs, we present the discrete forms respectively.

(1) Method I: EM scheme as (5.2).

(2) Method II: Milstein scheme

$$
\left\{\begin{array}{l}
x_{k+1}=x_{k}+\left(\sigma\left(-x_{k}+y_{k}\right)+0.5 \mu^{2} x_{k}\right) \Delta t_{k}+\mu x_{k} \Delta W_{k}+0.5 \mu^{2} x_{k}\left(\left(\Delta W_{k}\right)^{2}-\Delta t_{k}\right), \\
y_{k+1}=y_{k}+\left(-x_{k} z_{k}-\rho x_{k}+y_{k}+0.5 \mu^{2} y_{k}\right) \Delta t_{k}+\mu y_{k} \Delta W_{k}+0.5 \mu^{2} y_{k}\left(\left(\Delta W_{k}\right)^{2}-\Delta t_{k}\right), \\
z_{k+1}=z_{k}+\left(x_{k} y_{k}-\beta z_{k}+0.5 \mu^{2} z_{k}\right) \Delta t_{k}+\mu z_{k} \Delta W_{k}+0.5 \mu^{2} z_{k}\left(\left(\Delta W_{k}\right)^{2}-\Delta t_{k}\right) .
\end{array}\right.
$$

Tables 1 and 2 present the numerical results, where $\delta$ is the local error and $\varepsilon$ is the shadowing distance. They show the existence of shadowing orbit and the effectiveness of the numerical method. Because we choose $\left\{\hat{h}_{k}\right\}_{k=0}^{N}=\left\{t_{k}\right\}_{k=0}^{N}$, that is, we do not consider the reparameterization of time, the values of shadowing distance $\varepsilon$ are not small with respect to the local error $\delta$.

Table 1: Summaries of the parameters for stochastic Lorenz equations for EM scheme.

\begin{tabular}{cccccc}
\hline & EM & \multicolumn{5}{c}{$\mathrm{T}=10.5$} \\
\cline { 2 - 6 }$\mu$ & $\mathrm{c}$ & $\alpha$ & $\delta$ & $\beta$ & $\varepsilon$ \\
\hline 0 & 435.7302 & 1.4603 & $1.0500 \mathrm{e}-3$ & $8.7832 \mathrm{e}-4$ & 0.9181 \\
0.5 & 428.3332 & 11.1537 & 0.0324 & 0.0008 & 28.4786 \\
1.0 & 359.6973 & 80.1003 & 0.0324 & 0.0009 & 28.4989 \\
1.35 & 317.6131 & 126.1399 & 0.0324 & 0.0010 & 28.7522 \\
\hline
\end{tabular}

Table 2: Summaries of the parameters for stochastic Lorenz equations for Mistein scheme.

\begin{tabular}{cccccc}
\hline & Milstein & \multicolumn{4}{c}{$\mathrm{T}=10.5$} \\
\cline { 2 - 6 }$\mu$ & $\mathrm{c}$ & $\alpha$ & $\delta$ & $\beta$ & $\varepsilon$ \\
\hline 0 & 435.7302 & 1.4603 & $1.0500 \mathrm{e}-3$ & $8.7832 \mathrm{e}-4$ & 0.9181 \\
0.5 & 428.6890 & 10.1625 & $1.0500 \mathrm{e}-3$ & 0.0009 & 0.9216 \\
1.0 & 362.8804 & 79.6434 & $1.0500 \mathrm{e}-3$ & 0.0010 & 0.9293 \\
1.35 & 319.9973 & 126.0979 & $1.0500 \mathrm{e}-3$ & 0.0011 & 0.9368 \\
\hline
\end{tabular}

In Figure 3, we present the shadowing orbits of the $(\omega, \delta)$-pseudo orbits by the numerical computation with different numerical scheme. The first two figures are about the EM method, and the other two figures are about Milstein method. The blue line indicates the shadowing orbits and the red dots represent the $(\omega, \delta)$-pseudo orbits. As we show in this paper, the numerical realization of the $(\omega, \delta)$-pseudo orbits is shown as in Section 4, and the numerical realization of the shadowing orbits is obtained by the high order SDE-Taylor-like schemes. In fact, there is a differ in the accuracy of the numerical schemes. In order to show the detail cases which are shown in Tables 1 and 2, we only take the iterative step $N=1.5 e+3$. And the curve of the discrete shadowing orbits shows that oscillator and the relativity with the $(\omega, \delta)$-pseudo orbits. These phenomena mean that numerical experiment consists with the theory result of Theorem 3.7.

Finally, the approximative results of shadowing obtained from the shadowing method is shown in Figure 4 . The blue cycles indicate the shadowing orbits and the black stars represent the $(\omega, \delta)$-pseudo orbits. We only choose 10 points in the computational result. It presents that there are the shadowing orbits in the proper neighbourhood of the $(\omega, \delta)$-pseudo orbits. Therefore, the theoretical results can give information on chaotic orbits by the existence of shadowing orbits and the computation of shadowing distances. 

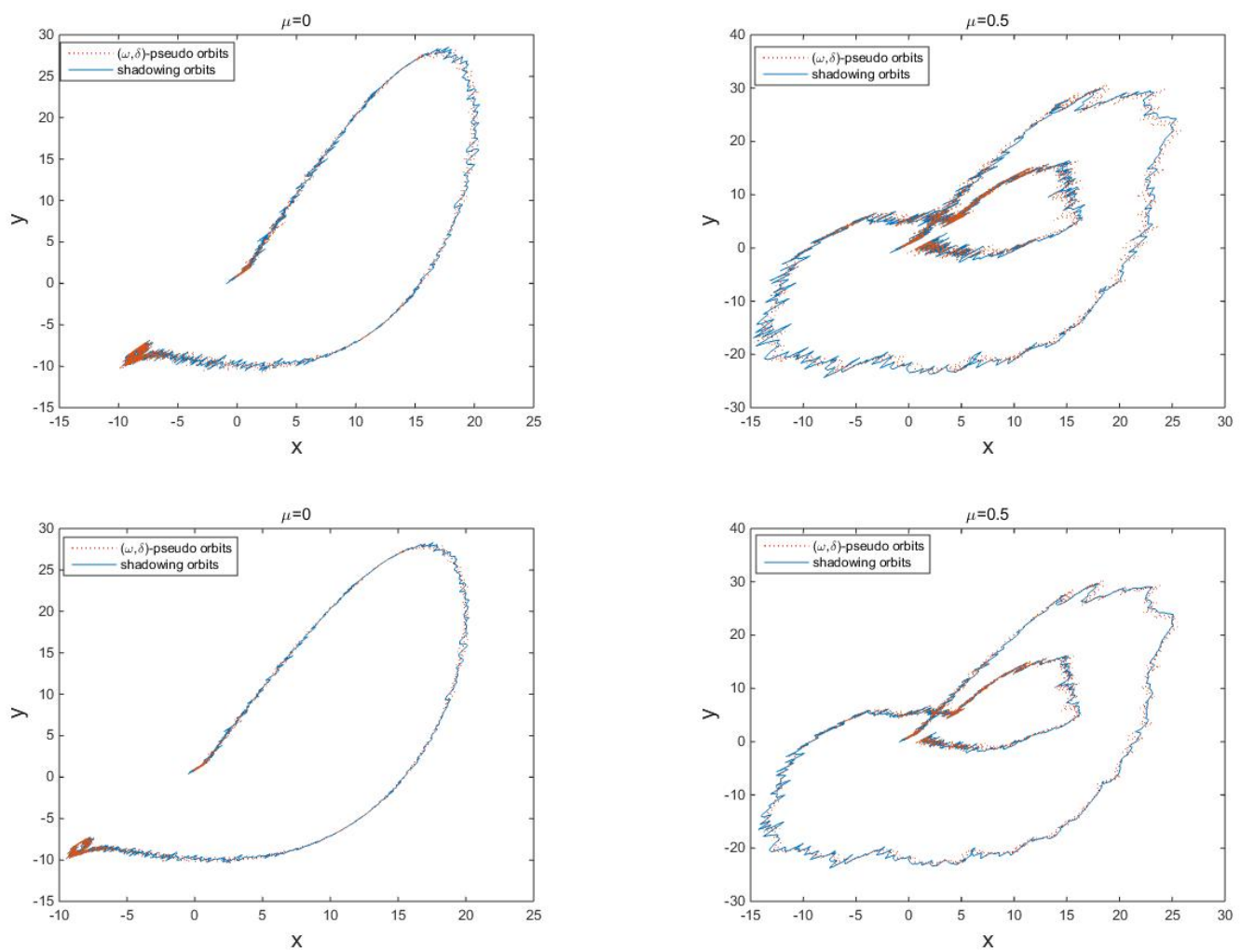

Figure 3: Qualitative graphs in parts showing experimental results in Table 1 for different methods and noises.

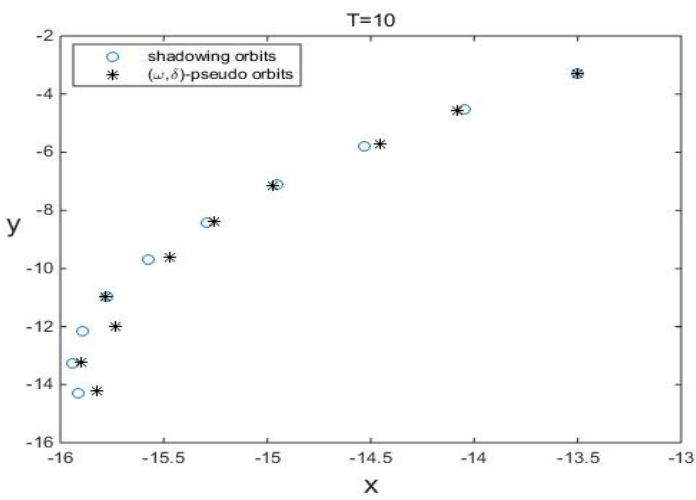

Figure 4: Qualitative graph in parts of the approximation shadowing trajectory on the stochastic Lorenz attractor.

As can be seen from these numerical results, there is an explicit dependent relationship between the shadowing distance and the local error, and there exists a true orbit in the appropriate neighborhood of an $(\omega, \delta)$-pseudo orbit of SDE (5.1). The numerically detected behavior of the system indeed reflects its real dynamical behavior. Furthermore, the higher the order of the scheme is, the shorter the shadowing distance will be.

\section{Conclusion}

Finally, conclusions and future work are summarized. In this paper, the main result is the shadowing theorem for finite time of SDE with explicit pseudo hyperbolic assumptions. This paper focuses on the feasibility and simulation of stochastic shadowing of a class of SDEs. The results show that the methods are effective and the numerical experiments are performed and match the results of theoretical analysis. 
Although some progresses are made, more simple and practical methods, and the explicit regularity conditions of SDEs, which can ensure the $(\omega, \varepsilon)$-shadowing and is also a huge project, will be shown in our further work.

\section{Acknowledgment}

Authors would like to express their gratitude to Prof. Xiangdong Xie for his constant supervision and support, and to Mr. Jialin Hong for his helpful discussions. We are indebted to the unknown referees whose suggestions improved the final version of the manuscript. This work is supported by NSFC (Nos. 11021101, 11290142, 91130003 and 91530118), the Fundamental Research Funds for the Central Universities, HUST, No. 2016YXMS226, The Natural Science Foundation of Fujian Province, No. 2015J01019, and Young Teacher's Education and Science Research Project in the Education Department of Fujian Province, No. JAT160182.

\section{References}

[1] L. Arnold, Random dynamical systems, Dynamical systems, Montecatini Terme, (1994), Lecture Notes in Math., Springer, Berlin, 1609 (1995), 1-43. 1, 2, 5.1

[2] L. Arnold, B. Schmalfuss, Lyapunov's second method for random dynamical systems, J. Differential Equations, 177 (2001), 235-265. 5.1

[3] N. D. Cong, Topological dynamics of random dynamical systems, Oxford Mathematical Monographs, The Clarendon Press, Oxford University Press, New York, (1997). 3.3

[4] J.-Q. Duan, An introduction to stochastic dynamics, Cambridge Texts in Applied Mathematics, Cambridge University Press, New York, (2015). 3.3

[5] A. Fakhari, A. Golmakani, Shadowing properties of random hyperbolic sets, Internat. J. Math., 23 (2012), 10 pages. 1

[6] C.-R. Feng, Y. Wu, H.-Z. Zhao, Anticipating random periodic solutions, I, SDEs with multiplicative linear noise, J. Funct. Anal., 271 (2016), 365-417. 3.2, 3.2

[7] G. H. Golub, C. F. Van Loan, Matrix computations, Fourth edition, Johns Hopkins Studies in the Mathematical Sciences, Johns Hopkins University Press, Baltimore, MD, (2013). 4

[8] L. V. Kantorovich, G. P. Akilov, Functional analysis, Translated from the Russian by Howard L. Silcock, Second edition, Pergamon Press, Oxford-Elmsford, N.Y., (1982). 3.3

[9] Y.-H. Li, Z. Brzeźniak, J.-Z. Zhou, Conceptual analysis and random attractor for dissipative random dynamical systems, Acta Math. Sci. Ser. B Engl. Ed., 28 (2008), 253-268. 1

[10] B. F. Liu, Y. L. Han, X. D. Sun, Square-mean almost periodic solutions for a class of stochastic integro-differential equations, (Chinese) J. Jilin Univ. Sci., 51 (2013), 393-397. 1

[11] G. N. Milstein, Numerical integration of stochastic differential equations, Translated and revised from the 1988 Russian original, Mathematics and its Applications, Kluwer Academic Publishers Group, Dordrecht, (1995). 4

[12] K. Palmer, Shadowing in dynamical systems, Theory and applications, Mathematics and its Applications, Kluwer Academic Publishers, Dordrecht, (2000). 1

[13] S. Y. Pilyugin, Shadowing in dynamical systems, Lecture Notes in Mathematics, Springer-Verlag, Berlin, (1999). 1

[14] T. Tél, M. Gruiz, Chaotic dynamics, An introduction based on classical mechanics, Translated from the 2002 Hungarian original by Katalin Kulacsy, Cambridge University Press, Cambridge, (2006). 1

[15] D. Todorov, Stochastic shadowing and stochastic stability, ArXiv, 2014 (2014), 16 pages. 1

[16] T.-C. Wang, Maximum norm error bound of a linearized difference scheme for a coupled nonlinear Schrödinger equations, J. Comp. Appl. Math., 235 (2011), 4237-4250. 1

[17] P. Wang, A-stable Runge-Kutta methods for stiff stochastic differential equations with multiplicative noise, Comput. Appl. Math., 34 (2014), 773-792. 1

[18] X.-D. Xie, Q.-Y. Zhan, Uniqueness of limit cycles for a class of cubic system with an invariant straight line, Nonlinear Anal., 70 (2009), 4217-4225. 1

[19] Q.-Y. Zhan, Mean-square numerical approximations to random periodic solutions of stochastic differential equations, Adv. Difference Equ., 2015 (2015), 17 pages. 2

[20] Q.-Y. Zhan, Shadowing orbits of stochastic differential equations, J. Nonlinear Sci. Appl., 9 (2016), 2006-2018. 1, 2, 3.1, 3.2, 3.3

[21] Q.-Y. Zhan, Y.-H. Li, Numerical random periodic shadowing orbits of a class of stochastic dierential equations, Chapter 10, Dynamical Systems-Analytical and Computational Techniques, edited by Mahmut Reyhanoglu, InTech, Rijeka, (2017). 1 